\title{
Control Proposals for the operation of Power Converters in Wind Power Systems
}

\author{
Alvaro Luna \\ Technical University of Catalonia \\ Electrical Engineering Department \\ Terrassa, Barcelona 08222 \\ Email: luna@ee.upc.edu
}

\section{Felipe Córcoles}

Technical University of Catalonia

Electrical Engineering Department

Terrassa, Barcelona 08222

Email: corcoles@ee.upc.edu

\author{
Daniel Aguilar \\ Technical University of Catalonia \\ Electrical Engineering Department \\ Terrassa, Barcelona 08222 \\ Email: danielg82_@hotmail.com
}

\author{
Raúl-S. Muñoz-Aguilar \\ Technical University of Catalonia \\ Electrical Engineering Department \\ Terrassa, Barcelona 08222 \\ Email: raul.munoz-aguilar@upc.edu
}

\begin{abstract}
The wind turbines based on Doubly Fed Induction Generators (DFIG) represent almost the $60 \%$ of the installed windpower worldwide. Nowadays most of the existing algorithms, used for regulating the production of active and reactive power in such systems, are based on control techniques that use rotational reference frames theories, where the $d q 0$ is maybe the most popular. Despite of its good operation under balanced conditions its behaviour depends on a good phase synchronization, otherwise the different transformations cannot be performed with enough guarantees. Of course this kind of response is becomming insuficient, as far as the windpower penetration is increasing. This paper will present a new control topology for DFIG, that are connected using back to back inverters, that is able to behave better in case of faulty grids. Besides some proposals for improving the operation of the DFIG will be presented as well. The aim of that part is to extend the possibilities that the back to back inverters are able to offer to the generator system by means of including new functionalities, as the active filtering capability or the reduction of the flicker emission that will be later discussed.
\end{abstract}

\section{INTRODUCTION}

The electric grid worldwide has been experiencing a very important evolution during the last years. The classical electricity infrastructure, that typically consist of large and centralized power generation facilities linked to the final consumers, is now turning into a distributed power generation system (Fig.1), where renewable energies are becoming important players.

In that scenario wind power systems have gained a great importance. During 2006 the cumulative wind power capacity operating in the EU increased by $19 \%$ and nowadays exceeds 48,000 MW. In an average wind year this will produce approximately $100 \mathrm{TWh}$ of electricity, equal to $3.3 \%$ of total EU electricity consumption [1]. Almost the $60 \%$ of this power is produced by doubly fed induction generators (DFIG) [2].

This kind of system belongs to the group of adjustable speed wind turbines. Although it is not the only one in that category, as there also topologies based on synchronous generators able
Pedro Rodríguez

Technical University of Catalonia

Electrical Engineering Department

Terrassa, Barcelona 08222

Email: prodriguez@ee.upc.edu

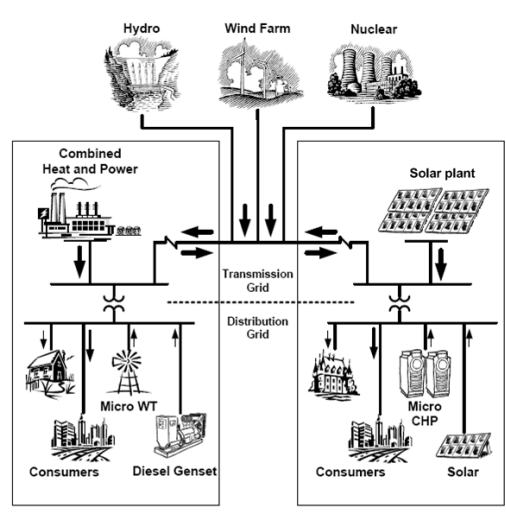

Fig. 1. Distributed power system model

to work is such conditions, it is the most extended one. This is due to the fact that it can be connected to the grid with no need of full power converters Therefore the cost of such wind turbines are lower, while the operation range (normally $25-30 \%$ of the nominal power) is broad enough in order to enhance its performance in front of fixed-speed generators [3].

In order to achieve these operation features the DFIG machine is connected to the grid using two converters in a back to back configuration, as it is presented in Fig. 2.

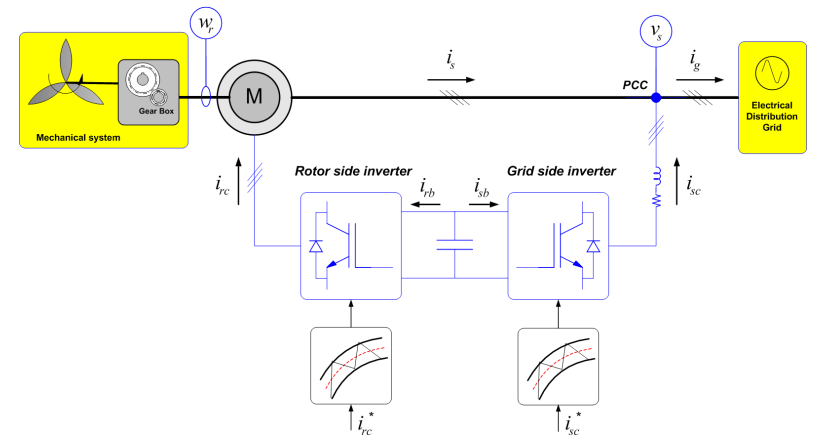

Fig. 2. DFIG operation using two converters in back to back configuration 
The classical operation technique of this system controls the active and reactive power that is delivered to the grid at the PCC thanks to the rotor side inverter, while the grid side one is responsible of maintaining the voltage level at the DC bus [4]. In this way a maximum power point tracking (MPPT) can be easily followed by the wind turbine, achieving thus an optimal performance of the generator.

This kind of operation has no problems when wind power systems are linked to a perfectly stable grid. Nevertheless the real network experiments voltage drops, frequency variations, phase jumps... that are even more important in weak grids, where wind farms are normally connected. This drawback was not so significant when the penetration of this kind of technology was still low, as there are protections able to disconnect the wind turbines in case of faults. But as far as they are becoming important energy producers their operation should be more robust [5].

This is a great challenge for wind power systems. Now it is not just a matter of injecting power into the grid, also themes regarding power quality should be taken into consideration. The aim of this paper is to propose control improvements in the current back to back topology for DFIG based wind turbines. These new control proposals intend to enhance the response of DFIG wind turbines in case of faults as well as integrating new functionalities in order to contribute to reinforce the power quality at the PCC. This is the case of the active filtering functionality, that will be later proposed, that extends the possibilities that the grid side power processor offers.

\section{DIRECT POWER CONTROL}

The traditional control designs normally consider the stator voltage as a set of three balanced sinewaves. Such systems can provide a good dynamic response during normal operation conditions, but the performance may be degraded in case of grid voltage disturbances, because this working hypothesis is then no longer valid.

Maybe the most extended method of controlling the injection of active $(\mathrm{P})$ and reactive power $(\mathrm{Q})$ in DFIG WT systems is the one proposed in [6]. In that paper a decoupled control of $\mathrm{P}$ and $\mathrm{Q}$ was presented, considering the basis of the classical control in the Park's reference frame [7] and introducing a decoupling network in order to improve the independent control of both variables. This algorithm requires, obviously, the transformation of stator and rotor voltage and currents, from $a b c$ to $d q 0$ reference frame, by means of the Park transforms.

The most relevant advantage of this control topology lies in the fact that conventional PI controllers can be used, yielding good performance. Nevertheless, the equations in Park's rotational reference frame depend strongly on a good synchronization with certain angles, for instance the synchronous reference needs to control the phase of the magnetic flux in order to obtain good results.

The proposed control topology layout of this paper is presented in Fig. 3. This system is able to deliver the currents that must be injected by the rotor and grid side power processors by means of measuring the mechanical speed of the rotor's shaft, and the currents and voltages at the PPC. Both magnitudes are represented by $i_{r c}^{*}$ and $i_{s c}^{*}$ respectively.

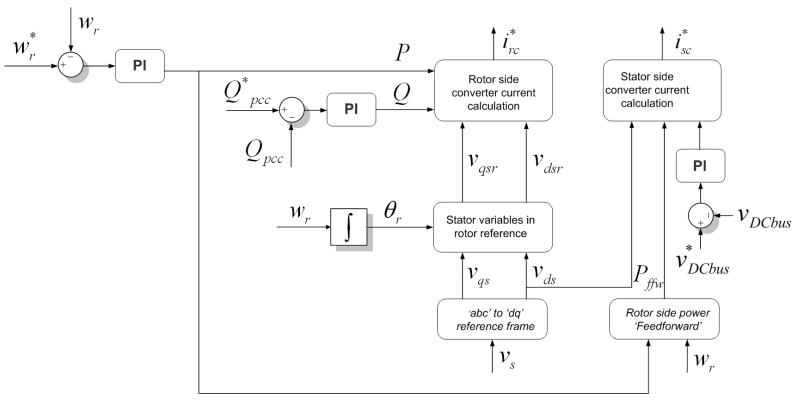

Fig. 3. DFIG control topology using an 'abc' reference frame

Later these set points are sent to current controllers, responsible of achieving the desired current at the output of the power processors. This operation can be performed using different methods, nevertheless none of them will be treated here, and both inverters will be considered current sources.

As it can be noticed the number of blocks in the control diagram is highly reduced, if compared with the ones that implement 'dq0' transformations, and hence the computational cost. In addition to that feature this new topology offer a more robust response, because its performance does not depend so much on a good synchronization with the grid voltage.

\section{A. Direct and inquadrature components}

The direct component of a three phase signal is equal to the input itself, thus just the identity matrix is necessary(1), as it is shown below where the grid voltage is represented in this reference,

$$
\begin{aligned}
& {\left[\overrightarrow{v_{s}^{d}}\right]=[I]\left[\overrightarrow{v_{s}}\right],} \\
& {\left[\begin{array}{c}
v_{a s}^{d} \\
v_{b s}^{d} \\
v_{c s}^{d}
\end{array}\right]=\left[\begin{array}{lll}
1 & 0 & 0 \\
0 & 1 & 0 \\
0 & 0 & 1
\end{array}\right] \cdot\left[\begin{array}{c}
v_{a s} \\
v_{b s} \\
v_{c s}
\end{array}\right] .}
\end{aligned}
$$

Considering that the input signal is a three phase vector, whose components are shifted $120^{\circ}$, a set of three inquadrature signals can be obtained by means of the following transformation,

$$
\begin{aligned}
& {\left[\overrightarrow{v_{s}^{q}}\right]=\frac{1}{\sqrt{3}} \cdot[M]\left[\overrightarrow{v_{s}}\right],} \\
& {\left[\begin{array}{c}
v_{a s}^{q} \\
v_{b s}^{q} \\
v_{c s}^{q}
\end{array}\right]=\frac{1}{\sqrt{3}} \cdot\left[\begin{array}{ccc}
0 & -1 & 1 \\
1 & 0 & -1 \\
-1 & 1 & 0
\end{array}\right] \cdot\left[\begin{array}{l}
v_{a s} \\
v_{b s} \\
v_{c s}
\end{array}\right] .}
\end{aligned}
$$

Both transformations (1) and (2) are only valid to define three phase systems with no homopolar sequence. This is due to an implicit condition that assumes that all three signals are shifted $120^{\circ}$. It could be demonstrated that all kind of 
situations can be represented using this new reference frame when there is a three phase - three wire grid. For doing that so, it is just necessary to remember that the positive and negative sequence are both a set of $120^{\circ}$ shifted three phase sine waves, so the direct and quadrature transformation can be applied in both cases.

It is worth to mention that the resulting inquadrature and direct components result in three phase sinusoidal signals, so no rotating reference frame is used here. Despite the fact that (1) and (2) are using the voltage as the base signal the same $I$ and $M$ matrix can be used for current vectors.

\section{B. Control of the rotor and grid side inverter}

The capability of producing inquadrature and direct signals permits to implement an easy method for calculating the current that the power processor has to deliver to the rotor in order to regulate the active and reactive power production/consumption of the WT [8],

$$
\begin{aligned}
& {\left[\overrightarrow{i_{r c}^{*}}\right]=\frac{P}{\left|\overrightarrow{v_{s r}^{d}}\right|^{2}} \cdot\left[\overrightarrow{v_{s r}^{d}}\right]+\frac{Q}{\left|\overrightarrow{v_{s r}^{d}}\right|^{2}} \cdot\left[\overrightarrow{v_{s r}^{q}}\right],} \\
& \left.\left|\overrightarrow{v_{s r}^{d}}\right|=\sqrt{\frac{2}{3}\left(\left(v_{a s r}^{d}\right)^{2}+\left(v_{b s r}^{d}\right)^{2}+\left(v_{c s r}^{d}\right)^{2}\right.}\right) .
\end{aligned}
$$

The expression (3) finds the current set point for the power processor, using the reference frame of the rotor. Thus the $\overrightarrow{v_{s r}^{d}}$ and $\overrightarrow{v_{s r}^{q}}$ vector can be obtained as follows,

$$
\begin{aligned}
& {\left[\overrightarrow{v_{s r}^{d}}\right]=\frac{2}{3} \cdot[\Gamma] \cdot\left[\overrightarrow{v_{s}^{d}}\right] \Rightarrow\left[\overrightarrow{v_{s r}^{d}}\right]=\left[\begin{array}{c}
v_{a s r}^{d} \\
v_{b s r}^{d} \\
v_{c s r}^{d}
\end{array}\right]} \\
& {\left[\overrightarrow{v_{s}^{d}}\right]=\left[\begin{array}{c}
v_{a s}^{d} \\
v_{b s}^{d} \\
v_{c s}^{d}
\end{array}\right]} \\
& {[\Gamma]=\left[\begin{array}{ccc}
\cos \theta_{r} & \cos \left(\theta_{r}-\frac{2 \pi}{3}\right) & \cos \left(\theta_{r}+\frac{2 \pi}{3}\right) \\
\cos \left(\theta_{r}+\frac{2 \pi}{3}\right) & \cos \theta_{r} & \cos \left(\theta_{r}-\frac{2 \pi}{3}\right) \\
\cos \left(\theta_{r}-\frac{2 \pi}{3}\right) & \cos \left(\theta_{r}+\frac{2 \pi}{3}\right) & \cos \theta_{r}
\end{array}\right],} \\
& {\left[\overrightarrow{v_{s r}^{q}}\right]=\frac{1}{\sqrt{3}} \cdot[M] \cdot\left[\overrightarrow{v_{s r}^{d}}\right]} \\
& {\left[\begin{array}{l}
v_{a s r}^{q} \\
v_{b s r}^{q} \\
v_{c s r}^{q}
\end{array}\right]=\frac{1}{\sqrt{3}} \cdot\left[\begin{array}{ccc}
0 & -1 & 1 \\
1 & 0 & -1 \\
-1 & 1 & 0
\end{array}\right] \cdot\left[\begin{array}{l}
v_{a s r}^{d} \\
v_{b s r}^{d} \\
v_{c s r}^{d}
\end{array}\right]}
\end{aligned}
$$

where $\theta_{r}$ is the rotation angle of the generator's rotor.

The resulting current at the stator side, when the rotor currents are determined in such way, regulates the active power thanks to the direct component, while the reactive power can be controlled by the inquadrature currents.

Regarding the current injection at the grid side just the feed forward of the power delivered by the rotor is needed in order to regulate the voltage at the DC link. For doing that so it is necessary to change the rotor power into stator power, thanks to the transformation,

$$
\begin{aligned}
& P_{f f w}=\frac{s}{s-1} \cdot P, \\
& s=\frac{w_{e}-w_{r}}{w_{e}} ; w_{e}=\frac{60 \cdot f}{p} .
\end{aligned}
$$

Finally, and thanks to the measured direct voltages at the PCC it is possible to state the currents that will be injected by the grid side power processor,

$$
\begin{aligned}
& {\left[\overrightarrow{i_{s c}^{*}}\right]=\frac{P_{f f w}}{\left|\overrightarrow{v_{s}^{d}}\right|^{2} \cdot\left[\overrightarrow{v_{s}^{d}}\right],}} \\
& \left|\overrightarrow{v_{s}^{d}}\right|=\sqrt{\frac{2}{3}\left(\left(v_{a s}^{d}\right)^{2}+\left(v_{b s}^{d} \cdot\right)^{2}+\left(v_{c s}^{d}\right)^{2}\right)} .
\end{aligned}
$$

The real reactive power can be experimentally measured by means of,

$$
\begin{aligned}
& Q_{p p c}=\overrightarrow{v_{s}} \times \overrightarrow{i_{g}} \\
& Q_{p p c}=v_{s a} \cdot\left(i_{b g}-i_{c g}\right)+v_{s b} \cdot\left(i_{c g}-i_{a g}\right)+v_{s c} \cdot\left(i_{a g}-i_{b g}\right) .
\end{aligned}
$$

The study of the stability when this method is used is not different from other already published [4]. In fact the 'dq0' components are useful in this part, because the stability of the system does not depend on the reference frame, therefore if the system is stable when rotational axis are used then it will be in any other circumstance.

\section{ACTIVE FILTERING FUNCTIONALITY}

In the classical control of DFIG systems the grid side inverter is just responsible of keeping a constant average voltage at the DC bus of the back to back power processor. However this front-end inverter is able to play a more important role in the control of the DFIG-WT. At least there is the chance of including further tasks to its normal operation, something that would permit to make the most of this hardware.

In this paper the inclusion of an active filter algorithm is proposed. This functionality would avoid the injection of harmonics into the stator of the DFIG, that would be performed by the grid side converter as it is shown in Fig. 4.

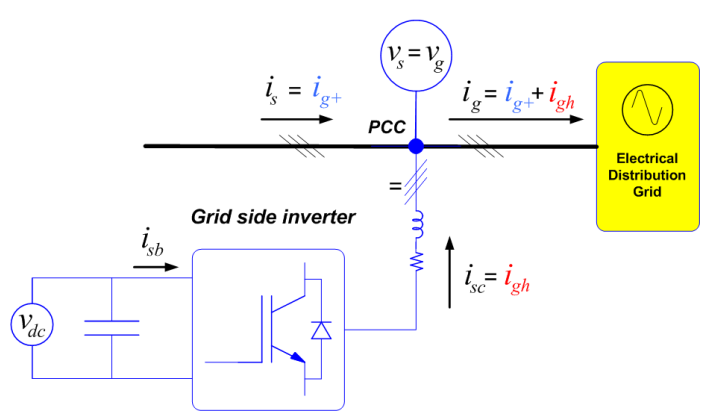

Fig. 4. Harmonic currents injection using the front-end power processor 
If the stator is free of harmonics the torque ripple in the shaft is lower, so the mechanical stresses are reduced. This feature is of great importance, because of the magnitude of the blades the mechanical stability is a critical point in this kind of generators. In addition other benefits could be obtained, for instance the magnetic flux can be easier estimated, because there is no oscillations (in case of using the classical P and Q control), therefore the control becomes easier.

This kind of application is able to coexist with the DC voltage control due to the fact that the harmonics currents produce no average energy variation at the capacitor. Therefore the active filter functionality could be performed in this case using the grid side inverter without interfering its current operation.

\section{A. Harmonic current reference}

In order to work as an active filter the front end converter needs to know the harmonic reference that must be injected to the PCC. Once this parameter is obtained it is introduced as a new set point to the current controller of the inverter (Fig.5), that has already another input for controlling the DC bus voltage.

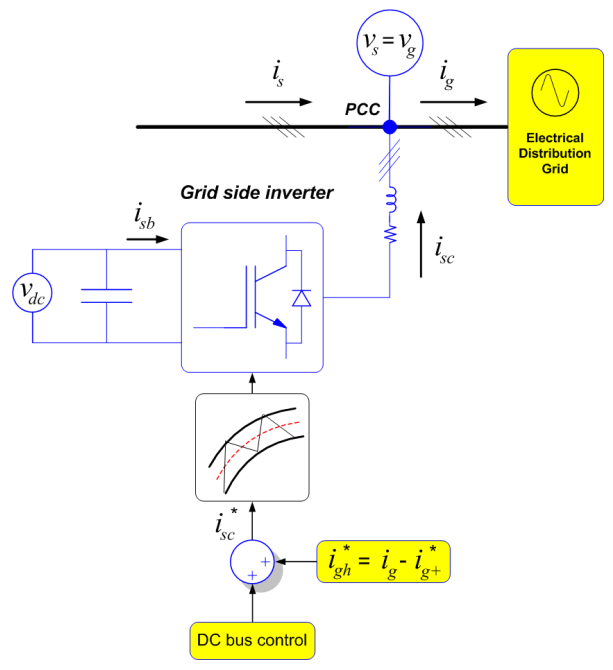

Fig. 5. Active filtering block diagram

The harmonic currents reference $\left(i_{g h}^{*}\right)$ can be found by means of subtracting the positive sequence $50 \mathrm{~Hz}$ current $\left(i_{g^{+}}^{*}\right)$, that is necessary for producing the required active power, from the total current that the generator is injecting to the grid $\left(i_{g}\right)$. Considering a balanced $50 \mathrm{~Hz}$ voltage grid the harmonic currents are those that doesn't change the mean value of the instantaneous active power of the system. This magnitude can be calculated as the dot product of the voltage and current at the PCC,

$$
\begin{aligned}
& p=\vec{v}_{g} \cdot \vec{i}_{g} \Rightarrow \vec{v}_{g}=\left[\begin{array}{c}
v_{g a} \\
v_{g b} \\
v_{g c}
\end{array}\right] ; \vec{i}_{g}=\left[\begin{array}{c}
i_{g a} \\
i_{g b} \\
i_{g c}
\end{array}\right], \\
& p=v_{g a} \cdot i_{g a}+v_{g b} \cdot i_{g b}+v_{g c} \cdot i_{g c} .
\end{aligned}
$$

The instantaneous active power can be expressed as the addition of a mean value, $\bar{p}$, and a oscillatory term $\tilde{p}$. The first one corresponds to the active power delivered by the system at the fundamental frequency. The value of this parameter can be easily extracted from $p$ using a simple lowpass filter,

$$
p=\bar{p}+\tilde{p}
$$

The $50 \mathrm{~Hz}$ positive sequence current vector that is necessary to produce the required active power ( (10) can be found thanks to (11). This equation calculate the magnitude of the current vector by means of dividing the mean value of the active power by the magnitude of the positive sequence voltage, while the direction of this variable is directly determined by the unitary vector of the positive sequence voltage vector,

$$
\vec{i}_{g^{+}}^{*}=\frac{\bar{p}}{\left|\vec{v}_{g^{+}}\right|^{2}} \cdot \vec{v}_{g^{+}} .
$$

Finally the harmonic set point is determined by subtracting the result of (11) from the measured grid current,

$$
i_{g h}^{*}=i_{g}-i_{g^{+}}^{*} .
$$

The resulting $i_{g h}^{*}$ will contain just harmonic currents if the voltage at the PCC has no negative sequence. If there is an unbalanced voltage this magnitude will contain also negative sequence currents. However this is not a drawback for the proposed filter, because this kind of currents act also as a torque disturbance for the machine, so it is better to deliver such components through the inverter. Nevertheless the injection of negative sequence currents can produce variations at the DC bus, but it can be easily regulated thanks to the DC bus voltage controller (Fig.5).

\section{Flicker attenuation}

In the DFIG based WT the control algorithms are designed in order to achieve the optimal active power generation. There are different techniques for doing that so, but maybe the most extended ones are the direct speed control and the direct active power control [9]. The first one implements a control loop where the rotational speed of the shaft is driven to the point where the power coefficient $(c p)$ of the machine reaches the maximum value. The direct power control performs the same control, but in an indirect way. This method offers an active power set point to the rotor side inverter depending on the rotational speed of the wind turbine. This value comes from the curve of MPPT that each WT present [10]. This algorithm finally converges to the optimal power production conditions, and indirectly is setting the speed of the machine.

If the speed or the power control is implemented with a fast controller the wind turbine performs a good average $c p$ value. Unfortunately this kind of operation transmits the wind oscillations to the generator, and as a consequence the active power presents a ripple at the PCC.

Low frequency power oscillations can create flicker at the PCC. Moreover this effect can be more noticeable in weak grids, due to the line impedance. Some authors have presented 
some solutions to this problem, like Sun et al. [11] that counteract the flicker emission by means of controlling the injection of reactive power to the grid. In this paper an easier proposal is presented. The inertia of the mechanical system is very high in the case of wind turbine, so it behaves as a great energy buffer. If the low frequency oscillations became a part of this mechanical energy then no power ripple would appear at the stator output. So it is just necessary to slow down the dynamics of the active power or the speed controller that operates the rotor side inverter. A soft control is able to take the changes in the mean value but rejects the oscillations of the wind that are conducted to the shaft of the blades.

Fig. 6 and 7 shows the influence of the power or speed controller. When a low frequency wind oscillation $(1 \mathrm{~Hz})$ is introduced the generated active power ripple increase if the controller has a fast response. On the contrary a soft controller almost eliminates the power ripple, as the variations of electrical energy goes down the speed of the turbine increases, acting as a buffer of energy.

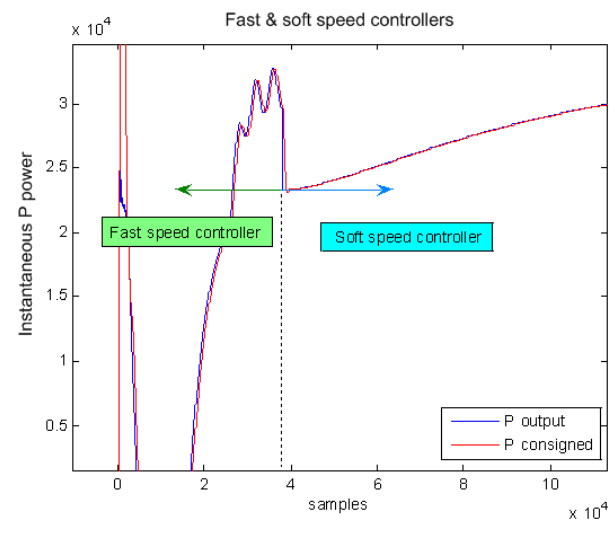

Fig. 6. Active power oscilation

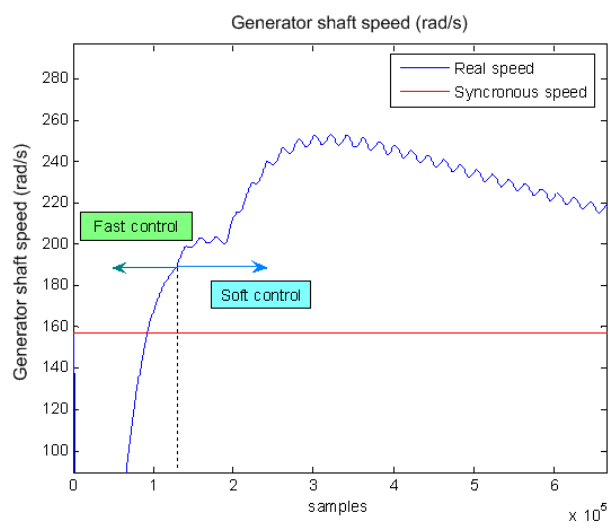

Fig. 7. Shaft speed oscilation

\section{CONCLUSION}

The present paper introduce new proposals for improving the performance of the current DFIG wind turbines that are linked to the grid by means of back to back inverters. A new power control philosophy for that topology, based on a ' $a b c^{\prime}$ reference frame, has been presented. This structure permits an easier control of the system, due to the fact that needs less mathematical operations. As a consequence the performance of the control becomes more robust, as it does not depend on any kind of synchronization or flux estimation. Therefore it performs a better response in case of faulty grids, where the operation of the WT results critical.

Besides, a new harmonic filtering functionality has been introduced in the classical back to back configuration. The front end power processor, that were just regulating the DC bus in the classical algorithms, is now able to act as an active filter at the same time. Both tasks are compatible, because the harmonic currents do not change the average value of the capacitor's voltage. The introduction of this algorithm improves the general behavior of the system, facilitating its control and also contributing to the enlarge the DFIGs life, because of the vibration reduction in the mechanical system. Finally some suggestions regarding the voltage flicker emission control have been also proposed. In that part it has been justified that the dynamics of the power control or speed control loop influence critically the response of the system in case of low frequency wind oscillations. Then if a soft controller is tuned the inertia of the the blades acts as a mechanical energy buffer where the torque oscillations can be stored, avoiding thus the emission of active power ripple that could finally produce flicker.

\section{ACKNOWLEDGMENT}

This work was supported by the Ministerio de Educacion y Ciencia of Spain under Project ENE2008-06588-C04-03/ALT

\section{REFERENCES}

[1] www.ewea.org, "Ewea aims for 22 of europe electricity by 2030," Wind Direction, 2006.

[2] L. Hansen, L. Helle, F. Blaabjerg, E. Ritchie, S. Munk-Nielsen, H. Bindner, P. Srensen, and B. Bak-Jensen, "Conceptual survey of generators and power electronics for wind turbines," Risoe, Tech. Rep., 2001.

[3] S. Muller, M. Deicke, and R. De Doncker, "Doubly fed induction generator systems for wind turbines," Industry Applications Magazine, IEEE, vol. 8, no. 3, pp. 26-33, May-June 2002.

[4] R. Pena, J. Clare, and G. Asher, "A doubly fed induction generator using back-to-back pwm converters supplying an isolated load from a variable speed wind turbine," in Electric Power Applications, IEE Proceedings-, vol. 143 , no. 5 , Sept. 1996 , pp. 380-387.

[5] e on, "Grid code - high and extra high voltage," www.eon-netz.com, April 2006.

[6] R. Pena, J. Clare, and G. Asher, "Doubly fed induction generator using back-to-back pwm converters and its application to variable-speed windenergy generation," in Electric Power Applications, IEE Proceedings-, vol. 143, no. 3, May 1996, pp. 231-241.

[7] P. Krause, O. Wasynczuk, and S. S., Analysis of Electric Machinery and Drive Systems second edition. Wiley-IEEE Press, 2002.

[8] G. Chang and T.-C. Shee, "A novel reference compensation current strategy for shunt active power filter control," Power Delivery, IEEE Transactions on, vol. 19, no. 4, pp. 1751-1758, 2004.

[9] T. Ackerman, Wind Power in Power Systems. John Wiley \& Sons, Ltd., 2005.

[10] S. Heier, Grid Integration of Wind Energy Conversion Systems. John Wiley \& Sons, 2005.

[11] T. Sun, Z. Chen, and F. Blaabjerg, "Flicker study on variable speed wind turbines with doubly fed induction generators," Energy Conversion, IEEE Transactions on, vol. 20, no. 4, pp. 896-905, Dec. 2005. 